\title{
A suction blister model reliably assesses skin barrier restoration and immune response
}

Tracey J. Smith, Marques A. Wilson, Andrew J. Young and Scott J. Montain: Military Nutrition Division, United States Army Research Institute of Environmental Medicine, Natick, MA

Skin wound healing models can be used to detect changes in immune function in response to interventions. This study used a test-retest format to assess the reliability of a skin suction blister procedure for quantitatively evaluating human immune function in repeated measures type studies. Up to eight suction blisters $\left(\sim 30 \mathrm{~mm}^{2}\right)$ were induced via suction on each participant's left and right forearm (randomized order; blister session 1 and 2), separated by approximately one week. Fluid was sampled from each blister, and the top layer of each blister was removed to reveal up to eight skin wounds. Fluid from each wound was collected 4, 7 and 24 hours after blisters were induced, and proinflammatory cytokines were measured. Transepidermal water loss (TEWL), to assess skin barrier recovery, was measured daily at each wound site until values were within $90 \%$ of baseline values (i.e., unbroken skin). Sleep, stress and inflammation (i.e., factors that affect wound healing and immune function), preceding the blister induction, were assessed via activity monitors (Actical, Philips Respironics, Murrysville, Pennsylvania), the Perceived Stress Scale (PSS) and C-reactive protein (CRP), respectively. Area-under-the-curve and TEWL, between blister session 1 and 2, were compared using Pearson correlations and partial correlations (controlling for average nightly sleep, PSS scores and CRP). The suction blister method was considered reliable for assessing immune response and skin barrier recovery if correlation coefficients reached 0.7 . Volunteers $(n=16 ; 12 \mathrm{M} ; 4 \mathrm{~F})$ were $23 \pm 5$ years [mean \pm SD]. Time to skin barrier restoration was $4.9 \pm 0.8$ and $4.8 \pm 0.9$ days for sessions 1 and 2 , respectively. Correlation coefficients for skin barrier restoration, IL-6, IL-8 and MIP-1 $1 \alpha$ were 0.9 $(\mathrm{P}<0.0001), 0.7(\mathrm{P}=0.008)$ and $0.9(\mathrm{P}<0.0001)$, respectively. When average nightly sleep, PSS scores and CRP (i.e., percent difference between sessions 1 and 2) were taken into consideration, 
correlations in immune response between sessions 1 and 2 were improved for IL-8 $(0.8$, $\mathrm{P}=0.002)$ and TNF- $\alpha(0.7, \mathrm{P}=0.02)$. The skin suction blister method is sufficiently reliable for assessing skin barrier restoration and immune responsiveness. This data can be used to determine sample sizes for cross-sectional or repeated-measures types of studies testing the impact of various stressors on immune response, and/or the efficacy of interventions to mitigate decrements in immune response to stress. 


\section{Introduction}

Immune responsiveness can degrade as a consequence of heightened physical and psychological stress associated with intense military training or operations, or similar scenarios in civilian populations (e.g., training to become a firefighter or police officer, or elite athletes). In the past, circulating blood markers of immune function were sampled to assess the systemic immune response. The results of various experiments indicate that immune responsiveness is suppressed in Warfighters exposed to physical and psychological stress during training and operational missions $(5 ; 9 ; 14)$. While immune markers from blood provide an indication of systemic immune function, they don't measure functional status (e.g., the ability to defend against a virus or heal from a wound) (4). In contrast, wound healing models directly assess functional status of the immune system, and provide insight into the innate immune system proinflammatory response, as well as tissue remodeling processes.

The suction blister model is an experimental procedure that was developed to study the immune response at a wound site (e.g. cytokine values), as well as skin barrier restoration (a proxy measure of wound healing rate). An acrylic template with circular cutouts is attached to the skin, and vacuum suction separates the epidermis from the dermis producing skin surface blisters. Epidermal fluid that collects under the stratum corneum is sampled from each blister, and the top layer of each blister is removed to reveal standardized skin wounds. Fluid from each wound is subsequently collected over the following 24-hrs to investigate cytokine content at the wound site; and, transepidermal water loss is measured daily until the skin barrier is restored. The skin serves to limit the movement of water in and out of the body; therefore, the premise of measuring transepidermal water loss to assess skin barrier restoration is based on the fact that skin damage increases water permeability, thus permeability decreases as the wound heals. The measurement of 
proinflammatory cytokines at the wound site during the early stages of wound healing, in addition to the measurement of skin barrier restoration, allows researchers to quantitatively assess human immune function.

The suction blister model has been used during in vivo human experiments to study how stress affects immune responsiveness $(4 ; 6)$ and the efficacy of stress counter-measures (13) (e.g., nutritional interventions). Glaser et al. reported that within-subject cytokine (IL1 $\alpha$, Il-8) response from fluid that was sampled from different wound sites, induced on the same day, was reproducible $(r>0.7)(4)$. However, the test-retest reliability of cytokine and transepidermal water loss measures, taking into account day-to-day within-subject variation has not been reported. Therefore, the primary aim of this study was to assess the reliability of the cytokine response and the time to skin barrier restoration in a skin wound produced with the suction blister model. We hypothesized that we would observe a moderate to high test-retest reliability of cytokine profiles and skin barrier restoration rates after inducing suction blisters in participant's left and right forearms, as indicated by correlation coefficients $(\mathrm{R})$ greater than or equal to 0.7 .

\section{Methods}

\section{Participants}

Participants were either military or civilian personnel assigned to Natick Soldier Systems Center, Natick, MA. Complete data was collected on 16 participants (September 2012 - March 2013). This sample size was based on a report by Fleiss (3), who indicated 15-20 subjects are sufficient for estimating the reliability of a quantitative variable. Study participation was strictly voluntary and no participant was required to participate. Each volunteer gave their written, informed consent after an oral explanation of the study. The study was approved by the 
Institutional Review Board, United States Army Research Institute of Environmental Medicine, Natick, MA.

Individuals were included if they were between the ages of 19 and 35 years, were generally healthy and not taking medications (including non-steroidal anti-inflammatory drugs and aspirin), were not pregnant or lactating, had no history of psychiatric disorder requiring hospitalization or psychiatric medication usage, and slept between 6.5 and 9 hours per night at least five days per week.

This was a randomized, cross-over study. Blisters were induced on the left and right forearm of each participant using a polycarbonate template (Neuroprobe, Cabin John, MD) and suction pump (KNF Lab Filtration Pump, Neuberger Inc., Trenton, NJ). Approximately one week elapsed between the blister-induction of contralateral limbs. Fluid was sampled from each blister, and the top layer of each blister was removed to reveal up to eight skin wounds. Fluid from each wound was collected at 4, 7 and 24 hours after blisters were induced to assess wound inflammatory responses. Transepidermal water loss (TEWL, to assess skin barrier function) was measured every $\sim 24$ hours after blister induction until the skin barrier was "restored" (i.e., when TEWL had recovered $90 \%$ of individual baseline values).

\section{Baseline Questionnaire}

Each participant completed a questionnaire to determine demographic information (age, sex, race, and ethnicity).

\section{Body Weight}

Body weight was measured in minimal clothing (shorts and t-shirt, without shoes) before each blister induction session using a calibrated electronically powered scale accurate to $0.1 \mathrm{~kg}$ (Tanita Digital Scale Model No WB-110A, Tokyo, Japan). This measurement confirmed weight 
stability between the two blister sessions (i.e. $\leq 1 \mathrm{~kg}$ difference), since inadequate energy intake and/or dehydration may alter the immune response.

\section{Assessment of General Sleep Patterns}

General sleep habits were assessed via the Morningness/Eveningness questionnaire, a paper-and-pencil sleep diary, and actigraphy. The Morningness/Eveningness questionnaire is a 19-item questionnaire that assesses general sleep habits (7). This questionnaire was used as an initial screener. If a participant had atypical sleep habits (indicated by scores lower than 31 or higher than 69), they were not included in any further testing. If a participant scored favorably on the morningness/eveningness questionnaire, they were asked to wear an actigraphy monitor (Actical, Philips Respironics, Murrysville, Pennsylvania), and record the time they went to bed and woke-up, for five days prior to the blister procedure in order to verify that they slept $\sim 6-9$ hours per night for the five nights immediately prior to inducing suction blisters.

\section{Assessment of Acute Life Stressors}

The Perceived Stress Scale (2) was administered on the morning of and prior to each blister induction session to assess acute life stressors that may have occurred between the two blister induction sessions. This scale is a 14-item, widely used, self-report measure of perceived stress. This measure, which has adequate reliability and validity, asks participants to rate how stressful they have found their life during the previous month (2). The items are answered on a 0 (never) to 4 (very) scale, with higher sum scores indicating greater perceived stress.

\section{Assessment of C-reactive Protein and Preparation of Autologous Serum for Blister Model}

\section{Procedure}

Prior to each blister induction, $\sim 12 \mathrm{~mL}$ of whole blood was drawn from an antecubital vein. The blood was processed to yield $\sim 3.5 \mathrm{~mL}$ of serum to be used in the autologous fluid 
mixture and for the assessment of C-reactive protein (CRP). The purpose of measuring CRP was to identify changes in inflammatory status between the two blister induction sessions.

\section{Suction Blister Induction and Fluid Sampling}

Blisters were created, in a randomized fashion, on the left and right forearm of each participant to assess test-retest reliability of the wound model. At least seven days separated blister induction on contralateral arms. This method has been used in several studies conducted at The Ohio State University $(4 ; 8)$ and the National Institute of Allergy and Infectious Diseases $(10 ; 11)$. A plastic template with circular cut-outs (Neuroprobe, Cabin John, MD) was attached to the skin, and a gentle suction (14 inches $\mathrm{Hg}$ or $6.88 \mathrm{psi}$ ) from a vacuum pump (KNF Lab Filtration Pump, Neuberger Inc., Trenton, NJ) separated the epidermis from the dermis producing a skin surface blister after approximately 90 minutes. A standard desk lamp (Advantus Corp, Jacksonville, FL) outfitted with a 1020 V, $100 \mathrm{~W}$ bulb was placed 12 inches from the blister induction device to encourage blister formation. After the formation of blisters, the area of each blister wound (i.e., diameter at the longest and shortest axis) was measured. Subsequently, blister fluid (i.e., epidermal fluid that collects under the stratum corneum) was sampled using a syringe, and analyzed for cytokine content. The blister roof was then removed using sterile forceps and scissors, and a multi-well template was attached to the skin. Each wound was bathed with approximately $1.0 \mathrm{~mL}$ of $30 \%$ autologous serum (i.e., $30 \%$ serum and $70 \%$ Hanks' balance salt solution) by syringing it into a small perforation at the top of each well (15). Autologous fluid was subsequently aspirated from 2-3 different wells at $~ 4,7$ and 24 hours post blistering, and fluid was analyzed for cytokine content (4).

\section{Cytokine Response}


Various cytokines were assessed from blister fluid and autologous wound fluid, including interleukin (IL)-6, tumor necrosis factor (TNF)- $\alpha$, IL-8, macrophage inflammatory protein (MIP) $1 \alpha$ and MIP1 $\beta$. Cytokines were analyzed, in duplicate, using Multiplex bead-based methods on Luminex ${ }^{\circledR}$ technology (Millipore, Billerica, MA). Fluid from each well was analyzed separately, and mean cytokine values were calculated for each time-point. Wells were excluded from calculations if less than $70 \%$ of autologous serum was recovered, in order to eliminate bias due to well leakage.

\section{Time to Skin Barrier Restoration}

The time to skin barrier restoration was assessed by measuring transepidermal water loss (TEWL) from each blister (VapoMeter, Delfin Technologies Inc., Stamford, CT). Per the manufacturer's suggestions and to avoid potential confounding external variables, measurements were taken at approximately the same time each day (i.e., morning), at least 2 hours after exercise and/or showering, and after acclimation to the room temperature and humidity for at least 30 minutes. TEWL was measured twice from each blister site and from a control site on the forearm, and the paired measurements were averaged to determine the average daily measurement and the average daily control, respectively. If paired values were not within $10 \%$ of each other, a third measurement was taken and the two closest values were averaged. Skin barrier measurements were discontinued when the average daily measurement minus the average daily control value reached the $90 \%$ recovery value as calculated in the equation below (8):

$90 \%$ Recovery $=[($ Average day 1 measurement - average day 1 control $) \times 0.10]$

Subject's average daily measurements were then exponentially regressed using their average daily TEWL value from Day 1 (24 hours post blistering) until the average daily measurement exceeded or met $90 \%$ of the original skin barrier to better identify the precise moment of skin barrier restoration. 


\section{Statistical Analysis}

Statistical analysis was conducted using the IBM SPSS statistical package version 19.0 (IBM Inc., Armonk, New York). A two-tailed "p" value of 0.05 was considered statistically significant. The dependent variables of primary interest were skin barrier restoration rate and cytokine concentrations from the autologous wound fluid. For each cytokine, area-under-the curve (AUC) values were calculated for each subject using data obtained from autologous wound fluid sampled from the right arm and left arm in the 24-hours following wound induction (time 0, 4-hr, 7-hr, and 24-hr). Briefly, 'Area under the curve with respect to ground' $\left(\mathrm{AUC}_{g}\right)(12)$ represents the total AUC for all measurements with consideration for the time difference between each measurement and their distance from baseline. In this equation, $m i$ represents the individual measurement and $n$ equals the total number of measurements.

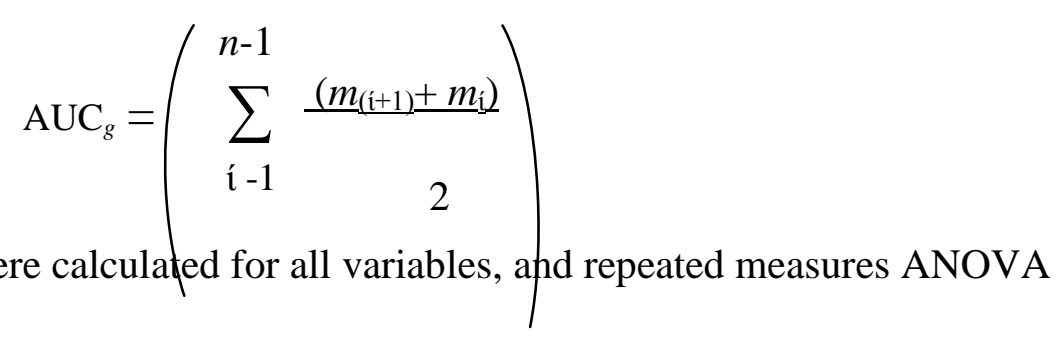

with Bonferonni correction determined significant differences over time for cytokines. Testretest reliability (session 1 vs. session 2) of 'time to skin barrier restoration' and cytokine response were examined using interclass (Pearson's) correlation coefficients. The suction blister model was considered reliable for measuring 'time to skin barrier restoration' and cytokine response if interclass correlation coefficients $(\mathrm{R})$ were greater or equal to 0.7 .

Correlation coefficients were also calculated for body weight, stress (i.e., PSS), inflammation (i.e., CRP), average sleep time prior to each blister session, suction time, and average area of each blister. If any of these variables were not significantly correlated between 
blister sessions, they were held constant using partial correlations when examining the test-retest reliability of 'time to skin barrier restoration' and cytokine response.

\section{Results and Discussion}

Volunteers (12 males and 4 females) were $23 \pm 5$ years and mainly White $(14$; black = 2) and non-Hispanic or Latino (14; Hispanic or Latino $=2$ ). Descriptive data and correlations between session 1 and session 2, for body weight, indicators of stress, average nightly sleep, suction time and blister area are displayed in Table 1.

Table 1: Demographic characteristics: descriptive statistics and correlations between blister session 1 and session 2

\begin{tabular}{|c|c|c|c|c|c|c|}
\hline \multirow[b]{2}{*}{ Variable } & \multicolumn{2}{|c|}{ Blister session 1} & \multicolumn{2}{|c|}{ Blister session 2} & \multicolumn{2}{|c|}{ Correlations } \\
\hline & Mean & $\begin{array}{l}\text { Standard } \\
\text { Deviation }\end{array}$ & Mean & $\begin{array}{l}\text { Standard } \\
\text { Deviation }\end{array}$ & $\mathrm{R}$ & P-value \\
\hline $\begin{array}{l}\text { Body weight } \\
(\mathrm{kg})\end{array}$ & 75.3 & 13.4 & 77.8 & 14.6 & 0.8 & 0.01 \\
\hline $\begin{array}{l}\text { Perceived } \\
\text { Stress Scale } \\
\text { score } \\
\text { (points) }\end{array}$ & 29.5 & 3.8 & 29.3 & 2.5 & 0.2 & 0.40 \\
\hline $\begin{array}{l}\text { C-reactive } \\
\text { protein } \\
(\mathrm{mg} / \mathrm{L})\end{array}$ & 1.8 & 2.2 & 2.9 & 2.8 & 0.1 & 0.70 \\
\hline $\begin{array}{l}\text { Average } \\
\text { nightly sleep } \\
(\mathrm{hrs})^{1}\end{array}$ & 7.2 & 0.8 & 7.2 & 0.7 & 0.2 & 0.50 \\
\hline $\begin{array}{l}\text { Suction time } \\
\text { (mins) }\end{array}$ & 98.1 & 13.0 & 96.4 & 15.7 & 0.5 & 0.04 \\
\hline $\begin{array}{l}\text { Blister area } \\
\left(\mathrm{mm}^{2}\right)\end{array}$ & 30.5 & 6.9 & 31.5 & 9.0 & 0.5 & 0.05 \\
\hline
\end{tabular}


As indicated in Table 1, CRP, average nightly sleep and PSS scores were not significantly correlated between session 1 and session 2 ; therefore, the test-retest reliability of ‘time to skin barrier restoration' and cytokine response was also examined after controlling for variations in these variables.

\section{Skin Barrier Restoration \& Test-Retest Reliability}

Time to skin barrier restoration was $4.9 \pm 0.8$ and $4.8 \pm 0.9$ days for session 1 and session 2 , respectively. The correlation coefficient for skin barrier restoration rate between blister session 1 and 2 was $0.7(\mathrm{P}=0.01)$. The relationship between blister sessions was not improved when average nightly sleep, CRP, and PSS scores were controlled for $(\mathrm{R}=0.7, \mathrm{P}=0.007)$.

The blister method has been employed by others to investigate the impact of stress and anger on wound healing $(4 ; 6)$; however, no published studies have assessed the within person, day-to-day variability in skin barrier restoration. The current study's findings indicate that skin barrier restoration rate is reliable as the relationship between suction blister wounds that were created on participant's left and right forearms on different days met the a priori goal (i.e. Rvalue greater or equal to 0.7 ). Further, skin barrier restoration was not sensitive to the within subject differences in average nightly sleep and stress that was encountered during the study.

\section{Cytokine Response \& Test-Retest Reliability}

Cytokine values from $\sim 15 \%$ of the wells were excluded from calculations, since less than $70 \%$ of autologous serum was recovered from these wells. Serial cytokine data for one subject was not collected after insufficient autologous serum was prepared during the suction procedure; and, data for three volunteers was excluded from all MIP1 $\alpha$ analyses due to their MIP1 $\alpha$ values falling more than 2 SDs from the mean The cytokine responses over time are shown in Figure 1. Almost all cytokines demonstrated a significant increase over time from baseline (pre-exposure 
$30 \%$ autologous serum) to 4-h and 7-h, with the exception of TNF $\alpha$ (blister session 2 only) and MIP1 $\alpha$ (both blister sessions), $p<0.01$. Therefore, cytokine data is presented as AUC from this point forward to capture the response over the full time-course from baseline to 24-h.

(insert Figure 1. Cytokine responses Blister Session 1 versus Blister Session 2)

The cytokine responses for each volunteer, expressed as AUC, are shown in Figure 2.

(insert Figure 2. Individual cytokine responses: Blister Session 1 versus Blister Session 2)

Area-under-the-curves (mean $\pm \mathrm{SD}$ ) and correlation coefficients, between session 1 and blister session 2, for all cytokines are listed in Table 2.

Table 2. Cytokine responses: descriptive statistics and correlations between blister session 1 and blister session 2

\begin{tabular}{|c|c|c|c|c|c|}
\hline & \multicolumn{2}{|c|}{ Blister session 1} & \multicolumn{2}{|c|}{ Blister session 2} & \multirow{3}{*}{$\begin{array}{c}\text { Correlation } \\
\text { R-value }\end{array}$} \\
\hline & \multicolumn{4}{|c|}{$\mathrm{AUCg}^{\mathrm{I}}$} & \\
\hline Cytokine & Mean & SD & Mean & SD & \\
\hline IL6 & 63544 & 56040 & 84248 & 78713 & $0.9 * * *$ \\
\hline IL8 & 133418 & 53233 & 138534 & 60022 & $0.7 * *$ \\
\hline TNF- $\alpha$ & 5253 & 4500 & 5665 & 3997 & 0.5 \\
\hline MIP1- $\alpha^{2}$ & 82234 & 75952 & 78160 & 72730 & $0.9 * * *$ \\
\hline MIP1- $\beta$ & 54519 & 43308 & 72096 & 55314 & 0.5 \\
\hline
\end{tabular}

${ }^{\mathrm{I}}$ Area-under-the-curve with respect to ground; represents the total AUC for all measurements with consideration for the time difference between each measurement and their distance from zero.

${ }^{2}$ data for three volunteers was excluded from the MIP $1 \alpha$ analyses due to their MIP $1 \alpha$ values falling more than 2 SDs from the mean

$* * * \mathrm{p}<0.0001, * * \mathrm{p}<0.01$

In the current study, IL-6, IL-8, and MIP1- $\alpha$ values post-blistering were significantly correlated between blister sessions 1 and 2, with IL-6, IL-8 and MIP1- $\alpha$ reaching acceptable $a$ priori $\mathrm{R}$-values $(\mathrm{R}>0.7)$. In contrast, TNF- $\alpha$ and MIP1- $\beta$ values were not well correlated, perhaps because MIP1- $\beta$, for example, is more variable within subjects compared to the other cytokines. In agreement, Agalliu et al. reported that plasma levels of MIP1- $\beta$ had poor within subject reproducibility in 17 women who were tested on different days (1). Future cross- 
sectional intervention studies, using the suction blister model to assess immune responsiveness, should take into account the within subject variability of each cytokine when calculating sample size in order to avoid a Type II error.

When the effect of stress and sleep were considered, the correlation coefficient was higher for TNF- $\alpha(\mathrm{R}=0.7, \mathrm{P}=0.02)$ and IL-8 $(\mathrm{R}=0.8, \mathrm{P}=0.002)$, but unchanged for the other cytokines measured. This finding, related to TNF- $\alpha$, is consistent with another study that used the suction blister model, wherein Kielcolt-Glaser et al (2005) demonstrated a down-regulation of TNF- $\alpha$ in response to a 30-min disagreement with their spouse. Future studies using the suction blister method to assess immune response should consider measuring potential confounders, like stress, and then accounting for them in subsequent analyses in an attempt to reduce within subject variability.

\section{Limitations}

The current study attempted to control for factors that may influence skin barrier restoration rate and immune response (e.g., average nightly sleep and recent stress), in order to minimize within subject variability. However, these factors were assessed by self-report, which may not have been accurate. Further, average nightly sleep was only verified in the five days leading up to each blister session. Also, the current study did not consider diet and other emotional traits (e.g., anger expression and negative affect) that may impact skin barrier restoration or immune response, and subsequent reliability. Nevertheless, the methods used in the current study were adequate to successfully demonstrate reproducibility of immune response markers and skin barrier restoration from a skin wound model employing a suction blister.

\section{Conclusion}


The skin suction blister method is sufficiently reliable for assessing skin barrier restoration and immune responsiveness, based on the a priori goal (i.e. R-value greater or equal to 0.7 ). The within and between subject variability data from this study can be used to determine sample sizes for repeated-measure and cross-sectional study designs, respectively, to investigate the impact of various stressors on immune response, and/or the efficacy of interventions to mitigate decrements in immune response to stress. Future studies using the suction blister model to assess wound healing time or immune response at the wound site should consider stress, sleep habits, diet and other emotional attributes since these variables could confound study results and/or explain findings.

\section{Disclaimer}

The opinions or assertions contained herein are the private views of the author(s) and are not to be construed as official or reflecting the views of the Army or the Department of Defense. Any citations of commercial organizations and trade names in this report do not constitute an official Department of the Army endorsement of approval of the products or Services of these organizations.

\section{Acknowledgments}

The authors wish to thank the individuals who participated in the study. This research was supported in part by an appointment to the Postgraduate Research Participation Program at the U.S. Army Research Institute of Environmental Medicine (USARIEM) administered by the Oak Ridge Institute for Science and Education through an interagency agreement between the U.S. Department of Energy and USARIEM. 


\section{Reference List}

1. Agalliu I, Xue X, Cushman C, Cornell E, Hsing A, Kaplan R, Anastos K, Rajpathak S and Ho G. Detectability and reproducibility of plasma levels of chemokines and soluble receptors. Results in Immunology 3: 79-84, 2013.

2. Cohen S, Kamarck T and Mermelstein R. A global measure of perceived stress. J Health Soc Behav 24: 385-396, 1983.

3. Fleiss JL. Reliability of Measurements. In: The Design and analysis of clinical experiments, New York: John Wiley and Sons, 1986, p. 2-31.

4. Glaser R, Kiecolt-Glaser JK, Marucha PT, MacCallum RC, Laskowski BF and Malarkey WB. Stress-related changes in proinflammatory cytokine production in wounds. Arch Gen Psychiatry 56: 450-456, 1999.

5. Gomez-Merino D, Drogou C, Chennaoui M, Tiollier E, Mathieu J and Guezennec CY. Effects of combined stress during intense training on cellular immunity, hormones and respiratory infections. Neuroimmunomodulation 12: 164-172, 2005.

6. Gouin JP, Kiecolt-Glaser JK, Malarkey WB and Glaser R. The influence of anger expression on wound healing. Brain Behav Immun 22: 699-708, 2008. 
7. Horne JA and Ostberg $\mathbf{O}$. A self-assessment questionnaire to determine morningnesseveningness in human circadian rhythms. Int J Chronobiol 4: 97-110, 1976.

8. Kiecolt-Glaser JK, Loving TJ, Stowell JR, Malarkey WB, Lemeshow S, Dickinson SL and Glaser R. Hostile marital interactions, proinflammatory cytokine production, and wound healing. Arch Gen Psychiatry 62: 1377-1384, 2005.

9. Kramer TR, Moore RJ, Shippee RL, Friedl KE, Martinez-Lopez L, Chan MM and Askew EW. Effects of food restriction in military training on T-lymphocyte responses. Int J Sports Med 18 Suppl 1: S84-S90, 1997.

10. Kuhns DB, DeCarlo E, Hawk DM and Gallin JI. Dynamics of the cellular and humoral components of the inflammatory response elicited in skin blisters in humans. J Clin Invest 89: 1734-1740, 1992.

11. Kuhns DB and Gallin JI. Increased cell-associated IL-8 in human exudative and A23187treated peripheral blood neutrophils. J Immunol 154: 6556-6562, 1995.

12. Matthews JN, Altman DG, Campbell MJ and Royston P. Analysis of serial measurements in medical research. BMJ 300: 230-235, 1990.

13. McDaniel JC, Belury M, Ahijevych $\mathbf{K}$ and Blakely W. Omega-3 fatty acids effect on wound healing. Wound Repair Regen 16: 337-345, 2008. 
14. Tiollier E, Gomez-Merino D, Burnat P, Jouanin JC, Bourrilhon C, Filaire E, Guezennec CY and Chennaoui M. Intense training: mucosal immunity and incidence of respiratory infections. Eur J Appl Physiol 93: 421-428, 2005.

15. Zimmerli W and Gallin JI. Monocytes accumulate on Rebuck skin window coverslips but not in skin chamber fluid. A comparative evaluation of two in vivo migration models. $J$ Immunol Methods 96: 11-17, 1987. 
IL-8

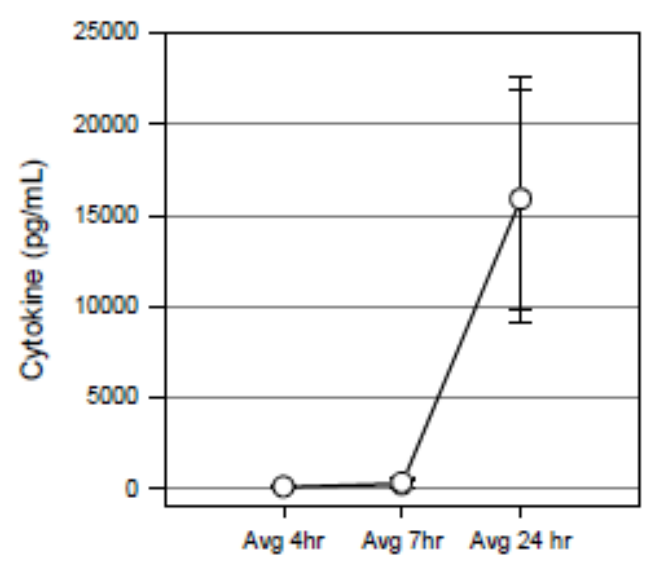

$\mathrm{TNF}-\alpha$

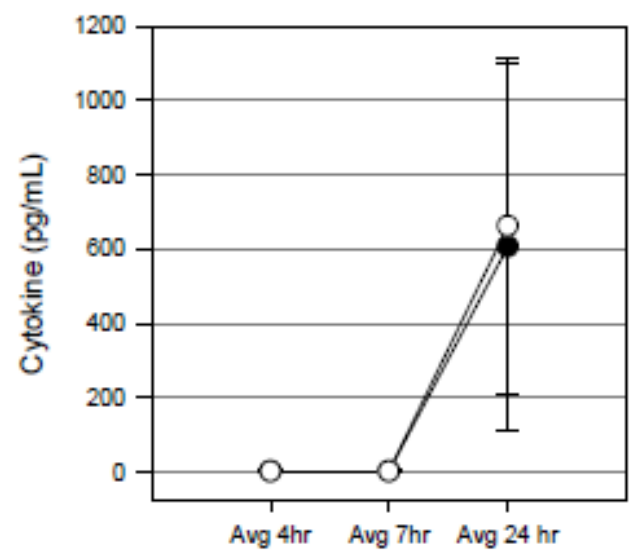

IL-6

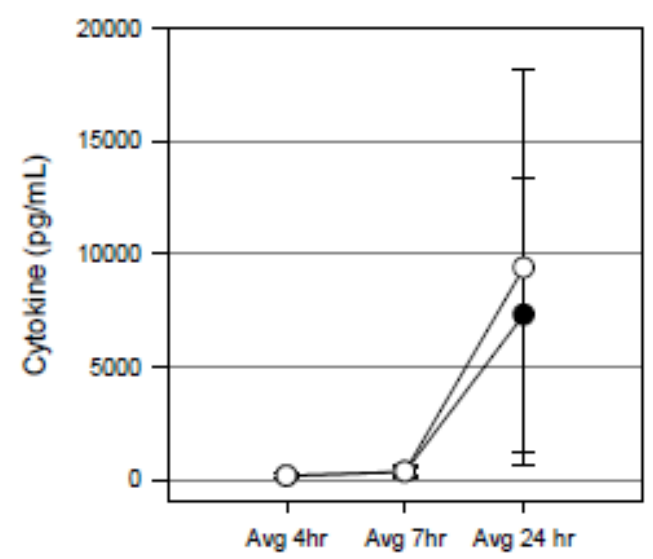

Figure 1: Cytokine Response Between Session 1 \& 2 . Note: $n=15$ for all ctyokines except MIP-1a, where $\mathrm{n}=12$ due to values falling more than 2 SDs from the mean for 3 participants.
MIP- $1 \alpha$

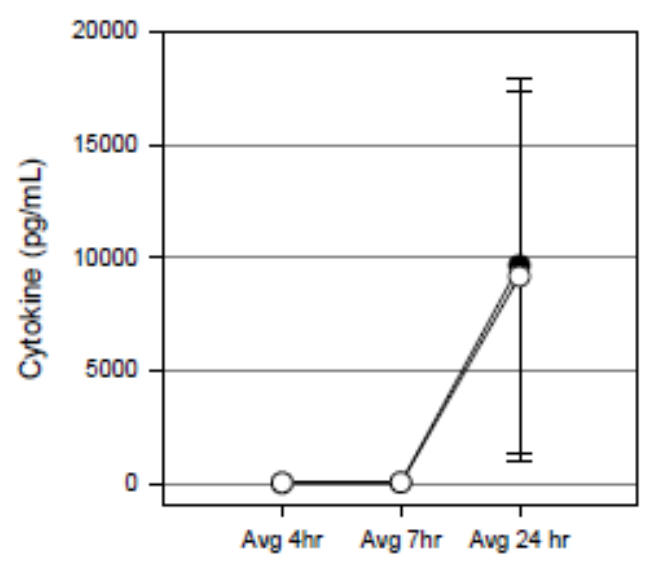

MIP-1 $\beta$

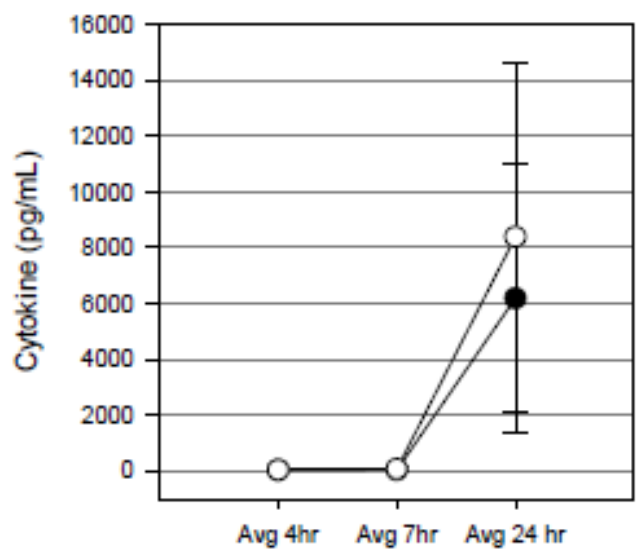


IL-8

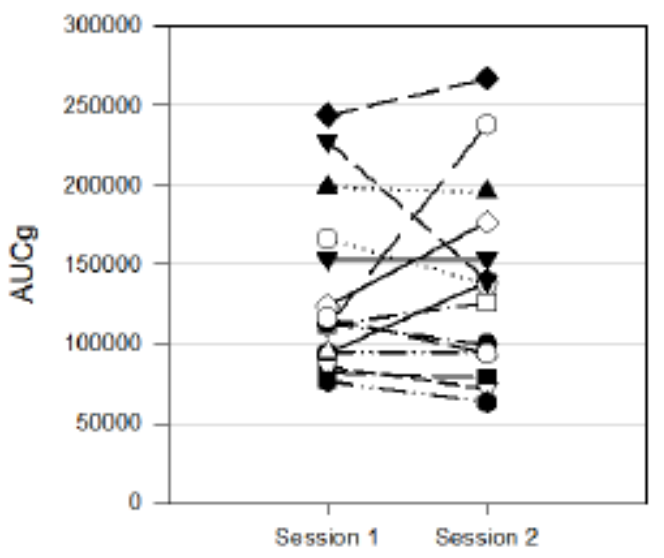

TNF- $\alpha$

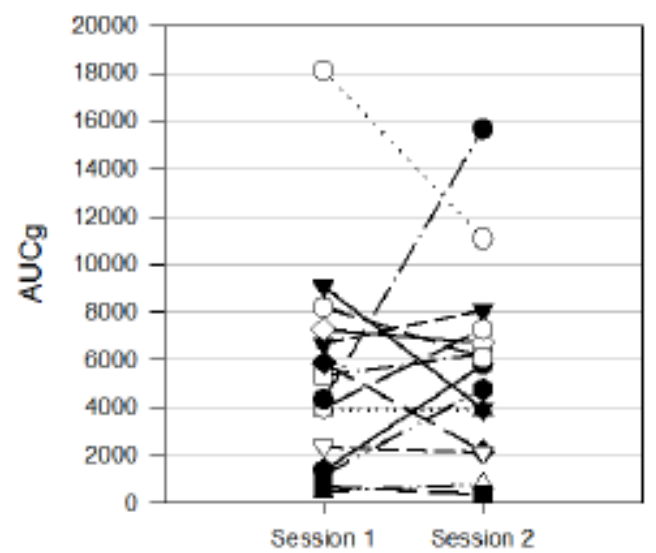

IL-6

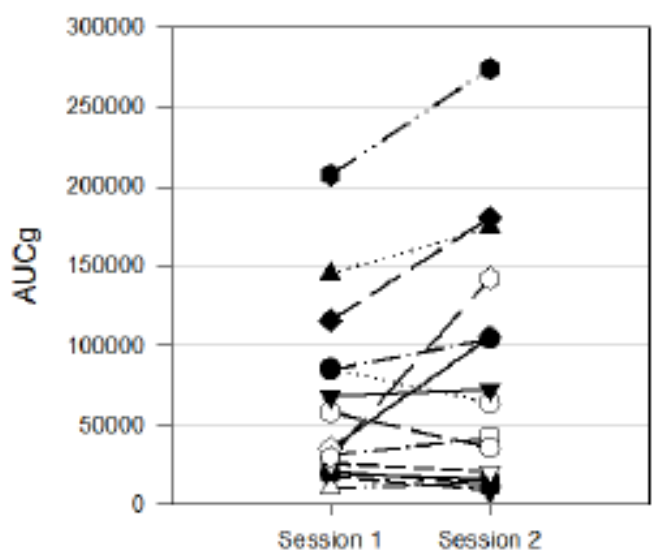

MIP-1 $\alpha$

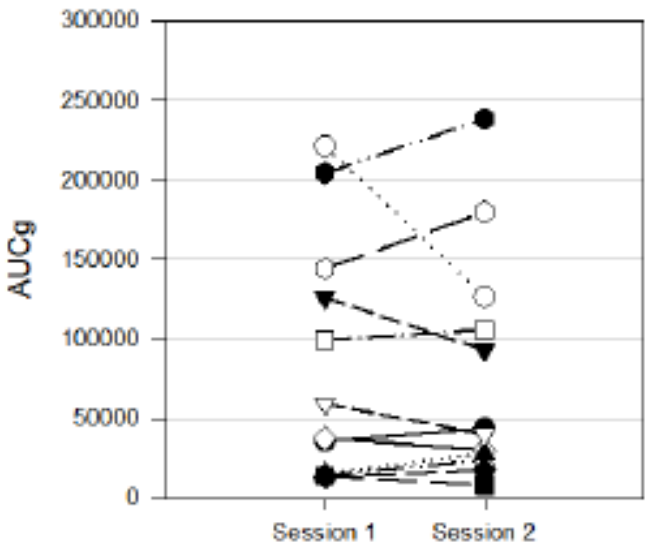

MIP-1B

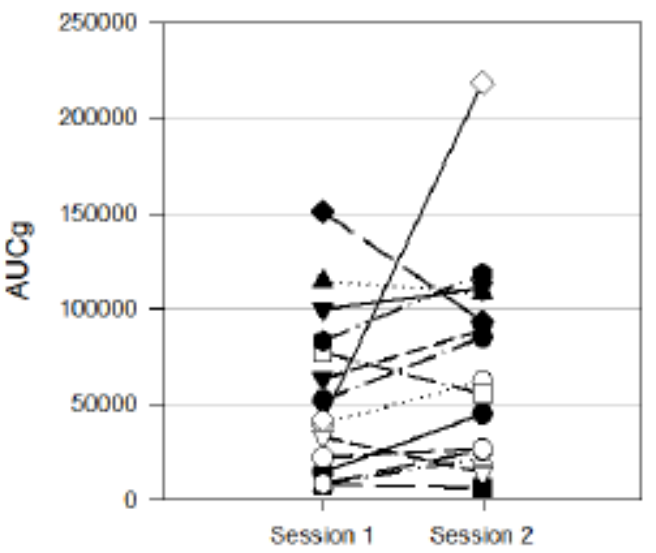

Figure 2: Cytokine Response Between Session 1 \& 2. Note: $n=15$ for all ctyokines except MIP-1a, where $\mathrm{n}=12$ due to values falling more than 2 SDs from the mean for 3 participants. 